\title{
Spectroscopic anatomy of a meteor with the very large telescope (ESO)
}

\author{
E. Jehin ${ }^{\text {a,* }}$, P. Jenniskens ${ }^{\text {b }}$, R.A. Cabanac ${ }^{\text {c }}$, C.O. Laux ${ }^{\text {d }}$, I.D. Boyd ${ }^{\mathrm{e}}$ \\ a European Southern Observatory (ESO), Casilla 19001, Santiago 19, Chile \\ b The SETI Institute, 2035 Landings Drive, Mountain View, CA 94043, USA \\ ${ }^{\mathrm{c}}$ Canada-France Hawaii Telescope (CFHT), Kamuela, HI 96743, USA \\ ${ }^{\mathrm{d}}$ Ecole Centrale Paris, Laboratoire EM2C, 92290 Chatenay-Malabry, France \\ e Department of Aerospace Engineering, University of Michigan, Ann Arbor, MI 48109-2140, USA
}

Received 1 March 2005; received in revised form 30 November 2006; accepted 4 December 2006

\begin{abstract}
A meteor spectrum was recorded serendipitously with the ESO Very Large Telescope during a long exposure in long-slit spectroscopic mode with the instrument FORS1. The -8 magnitude fireball crossed the narrow $(1 \operatorname{arcsec} \times 7$ arcmin $)$ slit during the observation of a high z supernova in normal service mode operation on May 12, 2002. The spectrum covers the range of 637-1050 nm, where the meteor's air plasma emissions from $\mathrm{N}_{2}, \mathrm{~N}$ and $\mathrm{O}$ dominate. The meteor trail appears moreover resolved along the slit but we conclude that this is because the meteor at $100 \mathrm{~km}$ altitude was out of focus for the VLT. The plasma excitation temperature varies only from about 4300 to $4365 \mathrm{~K}$ across the trail, based on the ratio of atomic and molecular nitrogen emissions. This is in agreement with the fact that the trail is not actually spatially resolved. Finally, carbon atom emission is not detected in the relatively unexplored range above 900 nm.
\end{abstract}

(C) 2006 COSPAR. Published by Elsevier Ltd. All rights reserved.

Keywords: Meteor spectroscopy; Meteor trail; Meteor temperature; Meteor composition

\section{Observation}

During a 20-min exposure in long-slit spectroscopic mode with the instrument FORS1 (FOcal Reducer and low dispersion Spectrograph) installed at the Cassegrain focus of Antu, one of the 8-m telescope of the European Southern Observatory Very Large Telescope (ESO VLT, Chile), an unusual signal was detected in an exposure starting at 03:01 UT May 12, 2002. The signal was only seen on the FORS1 spectrum and unfortunately there is no information regarding the orientation of the meteor path.

At the start of the exposure, the telescope was pointing at an elevation of $60.2^{\circ}$ (azimuth $11.8^{\circ}$ from North) at a mean airmass of 1.15 . The seeing was $1.4^{\prime \prime}$ (FWHM), measured from a star in the slit. The spectrograph was set up to have a slit $416.75^{\prime \prime}$ long and only $1.0^{\prime \prime}$ wide, with a position

\footnotetext{
${ }^{*}$ Corresponding author. Tel.: +56 2 4633054; fax: +56 24633001.

E-mail addresses: ejehin@eso.org (E. Jehin), cabanac@cfht.hawaii.edu (R.A. Cabanac).
}

angle of $178.7^{\circ}$ at the start of exposure $\left(\mathrm{N}=0^{\circ}, \mathrm{E}=90^{\circ}\right)$. The choice of grism 300I (see FORS usermanual at http://www.eso.org/instruments/fors1/), resulted in a dispersion of $108 \AA / \mathrm{mm}$ or $0.259 \mathrm{~nm} /$ pixel or a spectral resolution $\lambda / \Delta \lambda=660$ at the central wavelength of $860 \mathrm{~nm}$.

A zoom of the frame containing the anomalous signal is shown in Fig. 1, both before (a) and after (b) subtraction of the sky background. The spatial extension of the spectrum is about 150 pixels or $30^{\prime \prime}$ (pixel scale $=0.2^{\prime \prime} /$ pix.).

\section{Data reduction}

The following procedure was used to reduce the data with the IRAF ("Image Reduction and Analysis Facility") software package (NOAO). After subtraction of the CCD bias, the image was divided by a normalized flat field to correct for pixel-to-pixel sensitivity variations. A two-dimensional wavelength calibration was made, using a ThArHe lamp, to remove the curvature of the lines. The sky background was subtracted by fitting a 2 nd order 


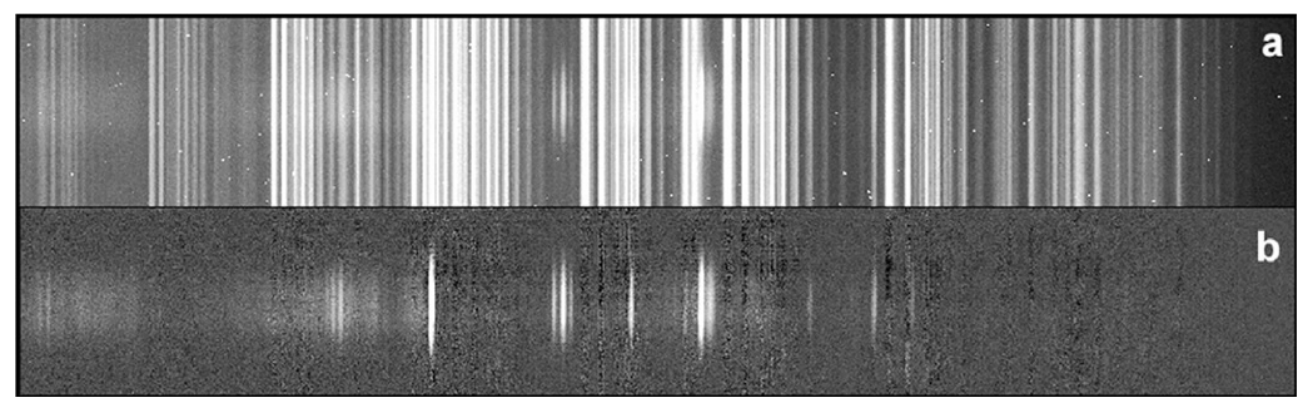

Fig. 1. Snapshots of the region of CCD frame showing the unusual signal: (a) with the sky displaying a lot of emission lines; (b) after a proper sky subtraction (see text).

Chebychev polynomial to two windows located just above and below the object. A one dimensional spectrum was extracted (see Fig. 2), with rejection of cosmic rays. The result was flux-calibrated using the response to a spectrophotometric standard star and taking into account the extinction for the altitude of the ESO Paranal Observatory and the airmass.

\section{Identification of lines and bands}

The unusual emission (Fig. 2) is clearly identified as originating from a meteor. At longer wavelengths than $600 \mathrm{~nm}$, meteor emission is usually dominated by air plasma emissions from $\mathrm{N}_{2}, \mathrm{~N}$ and $\mathrm{O}$. Atomic lines of $\mathrm{N}, \mathrm{O}$ and the First positive $\mathrm{B} 3 \Pi \mathrm{g} \rightarrow \mathrm{A} 3 \mathrm{\Sigma} \mathrm{u}+$ bands of $\mathrm{N}_{2}$ are present, which have relatively high upper state energies of $7-12 \mathrm{eV}$.

We checked for possible neutral and single ionized metal atom line emissions ( $\mathrm{Mg}$ I at $880-\mathrm{nm}, \mathrm{K}$ I doublet at $\sim 768$ $\mathrm{nm}$ ), but none was found. Indeed, all the new atomic lines in the ESO VLT meteor were identified as either oxygen or nitrogen atomic emissions (for a list of them see Jenniskens et al., 2004a).

\section{Altitude, speed and mass of the meteoroid}

A shower identification would establish the velocity vector of the meteor. The most active (1-2 meteors/h) among many minor showers is the Southern May Ophiuchid show- er (at $34 \mathrm{~km} / \mathrm{s})$, moving at an angle of a $\sim 64^{\circ}$ to the slit $\left(90^{\circ}\right.$ being perpendicular). However, lacking evidence for such a shower association this meteor may have had any possible orientation between slit and meteor path (angle $\alpha$ ) and speeds between 11 and $72 \mathrm{~km} / \mathrm{s}$.

If we use the Southern May Ophiuchid shower as an example, we find that the meteor would have been at an altitude of about $90 \mathrm{~km}(80-110 \mathrm{~km})$, or a distance of about $104 \mathrm{~km}$. The angular speed across the slit of the spectrograph would have been about $13.7 \%$ s and the slit crossing time would have been only $2.3 \times 10^{-5} \mathrm{~s}$.

The flux of the spectrum has been normalized to a continuum source and corresponds to an absolute (at a distance of $100 \mathrm{~km})$ magnitude of $-7.8\left(\sim 4.7 \times 10^{-13} \mathrm{~W} \mathrm{~cm}^{-2} \AA^{-1}\right)$ over the range [637-1050 nm]. If the meteor moved at a shallow angle of only say $20^{\circ}$ to the slit, then the meteor would have been one magnitude fainter. The meteor could have been a factor of two faster, which would change our estimate a factor of two brighter. In summary, we estimate that the meteor while crossing the slit had a mean brightness of $-7.8 \pm 1.1 \mathrm{mag}$.

\section{The meteor cross-section}

To our knowledge, this spectrum was the first to permit a direct and precise measurement of the initial radius of a meteor and the distribution of light in the trail (but see below). The distribution of light along the slit is found to
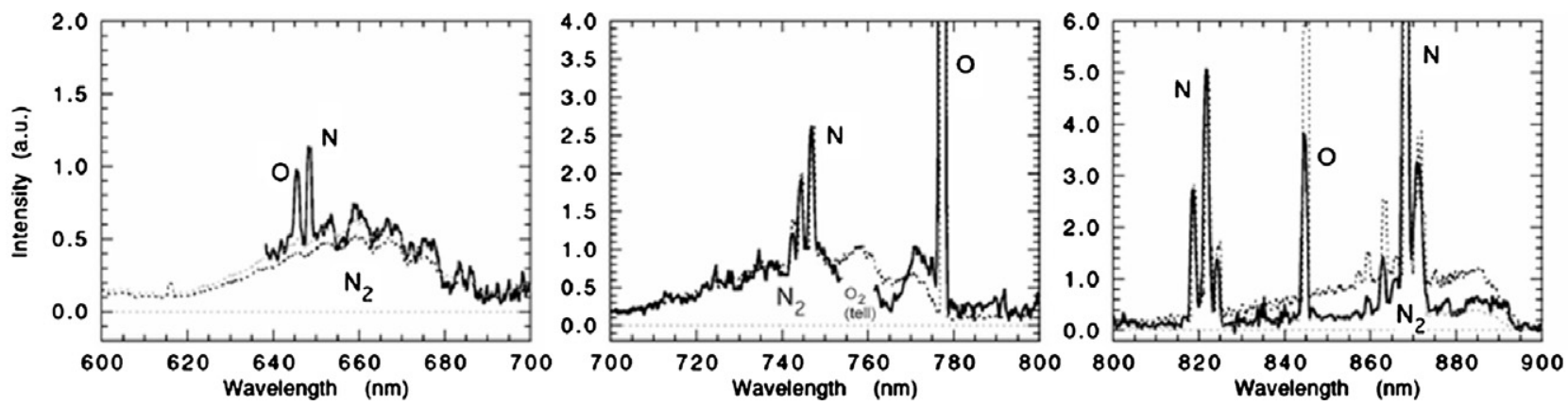

Fig. 2. Details of the VLT meteor spectrum (solid line). The dashed line is a NEQAIR2 model LTE air plasma spectrum, relevant for $95 \mathrm{~km}$ altitude $\left(P=10^{-6} \mathrm{~atm}\right)$ and $\mathrm{T}=4340 \mathrm{~K}$. Note that the model underpredicts the intensity of the $\Delta v=+3$ band series of $\mathrm{N}_{2}$ (left), while overpredicting the $\Delta v=+1$ band series (right). Thin dashed lines show the model of each individual band scaled to the observed band strength (in arbitrary units). 
be nearly Gaussian, with a measured FWHM $=14^{\prime \prime}$. This is $10 \times$ larger than the seeing $\left(1.4^{\prime \prime}\right)$ and resolves the volume of meteoric plasma in the direction along the slit.

We have $\mathrm{FWHM}=7.0( \pm 0.4) * \sin (\alpha) *(H(\mathrm{~km}) / 90)$ meter, where $\alpha$ is the unknown angle between the orientations of slit and meteor and the numerical value applies for an assumed altitude of $H=90 \mathrm{~km}$. This width is thought to reflect the mean-free path in the ambient atmosphere and should not depend on meteoroid mass or speed, as long as the meteoroid is in the rarefied flow regime.

Earlier, measurements were obtained from photographic traces, finding dimensions of 1-6 $\mathrm{m}$ for altitudes around 90-100 km (Cook et al., 1959, 1962 and ref.). The accuracy of these measurements was usually poor, because the trail width was significantly blurred by seeing and instrumental properties. Our result would be in agreement if the meteor crossed the slit at a reasonably tolerant $\alpha$ of $8^{\circ}-58^{\circ}$. Experimental data from radar amplitudes of underdense trails show an increase of the initial trail diameter from $2.74 \mathrm{~m}$ at $92 \mathrm{~km}$ to $8.14 \mathrm{~m}$ at $112 \mathrm{~km}$ for speeds of $34 \mathrm{~km} / \mathrm{s}$ (Baggaley, 1970, 1980, 1981; Ceplecha et al., 1998). This would suggest an altitude less than $\sim 108 \mathrm{~km}$.

\section{Temperature across the meteor trail}

Although predictions for the meteor trail width are in general agreement with the observations, the shape of the profiles predicted by models is not, making our observations very valuable.

The kinetic temperature cannot be measured directly from the Doppler width of the lines because the instrumental apparatus function is larger than a typical Dopplerbroadened line. However, there are several indirect measures that manifest themselves in the shape of the spectrum. Most importantly, a radial decrease of the kinetic energy will translate into a radial decrease of the ratio $\mathrm{N} /$ $\mathrm{N}_{2}$ and $\mathrm{O} / \mathrm{N}_{2}$ in the plasma. This is mainly because an increase of plasma temperature leads to a rapid decrease in the mole fraction of $\mathrm{N}_{2}$ (Jenniskens et al., 2000, 2004b).
Fig. 3 shows the variation of the ratios $\mathrm{N} / \mathrm{N}_{2}$ and $\mathrm{O} / \mathrm{N}_{2}$ along the slit. The remarkable result is that the $\mathrm{N} / \mathrm{N}_{2}$ ratio varies by only $27 \%$ and the $\mathrm{O} / \mathrm{N}_{2}$ ratio by only $11 \%$ across the trail. This variation indicates that the temperature gradient is small. The corresponding LTE (Local Thermodynamic Equilibrium) plasma temperature that would produce such an $\mathrm{N} / \mathrm{N}_{2}$ ratio is $\mathrm{T}=4340 \pm 20 \mathrm{~K}$ at the trail center, and varies only from 4300 to $4365 \mathrm{~K}$ across the trail. At the trail edge, there is no significant change of temperature on the scale of the train width.

With no variation in excitation temperature, the distribution of light should match closely the distribution of collisions in the meteor wake, integrated along the meteor path. About 3\% of the thermal collisions are thought to be inelastic (Öpik, 1958), leading to optical emissions. The volume-averaged collision rates calculated by Boyd (2000) were averaged along the meteor path and the line of sight, and compared in Fig. 3 with the meteor trail cross-section.

Curve (c) shows the expected line profile if light emission occurs only for energetic collisions of $\mathrm{N}_{2}$ and metal atoms with the ambient atmosphere, calculated by including a term $\exp \left(-E_{\mathrm{a}} / k T\right)$, with $E_{\mathrm{a}}=7.2 \mathrm{eV}$ and $T$ is the calculated gas temperature profile. We conclude that the observed trail is much wider than expected from current meteor expansion models, when taking into account the observed gas temperature profile.

If this broadening is due to emission being from higher altitude $\left(\alpha=90^{\circ}\right)$, then this would demand an unreasonable altitude $(>200 \mathrm{~km})$. If the broadening is due to a smaller angle $\alpha$, this demands a very improbable small angle of $3^{\circ}$ (for $90 \mathrm{~km}$ ), with the meteor moving nearly along the spectrograph slit. More importantly, such small angle alone does not explain the observed temperature profile and other processes are needed.

Since the meteor velocity (for almost all meteors) exceeds the Alfven critical velocity (which, for air is somewhere between 9 and $12 \mathrm{~km} / \mathrm{s}$ ), it is to be expected that the air along the meteor path will be strongly ionized (Zinn
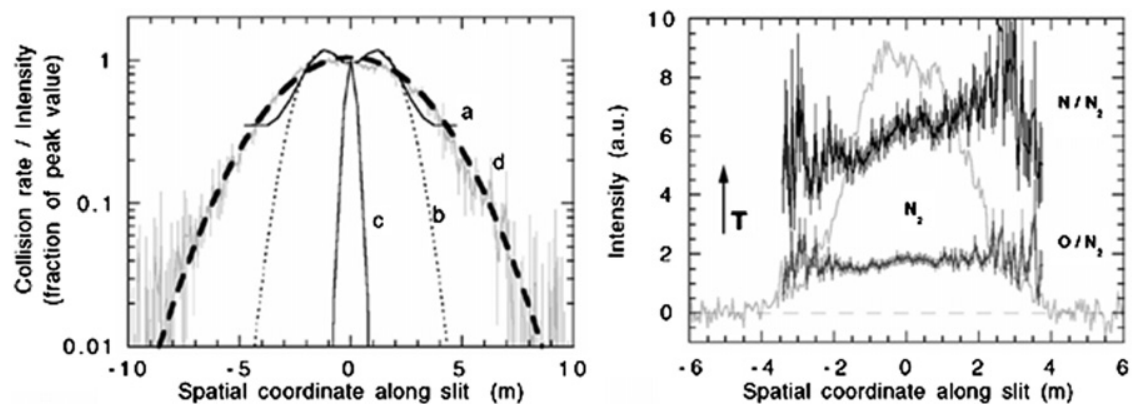

Fig. 3. On the left panel the meteor trail cross-section is expressed as the average intensity of N I and O I lines and the $\mathrm{N}_{2}$ molecular band along the slit. It is compared to a Direct Monte Carlo Simulation of the volume-averaged collision rate of a 1-cm size Leonid at $95 \mathrm{~km}$ altitude (Boyd, 2000), integrated along the meteor path and the line of sight, as a function of the third spatial coordinate along the slit. Curve (a) shows the collision rate of all molecules including those of the ambient air, curve (b) shows the result without ambient air molecules, while curve (c) shows the expected profile if light emission occurs only for energetic collisions with $\mathrm{N}_{2}$. Curve (d) - dashed curve - is a scaled version of curve (c) to the observed line profile. Right panel: ratio of N/ $\mathrm{N}_{2}$ and $\mathrm{O} / \mathrm{N}_{2}$ intensities along the length of the slit from top (negative) to bottom (positive). The nitrogen band intensity profile is shown for comparison. The line ratios increase with temperature, when a higher fraction of $\mathrm{N}_{2}$ is dissociated. 
et al., 2004) and that it, along with the meteor vapors, will emit strongly in the ultraviolet, resulting in dissociation of surrounding air out to considerable distances from the meteor track (Stenbaek-Nielsen and Jenniskens, 2004). This process is largely unrelated to LTE, as are the subsequent chemical reactions, which tend gradually to restore the system toward equilibrium. Therefore, it does not make sense to infer a temperature from the ratios of observed intensities of atomic and molecular emissions.

On the other hand, the observed ratio of atomic and molecular emissions are as expected for an LTE plasma with a plasma temperature derived from the metal atom line ratios (Jenniskens et al., 2004b). We also do not observe the expected strong $\mathrm{N}_{2}+$ emission in our spectrum (see also: Jenniskens et al., 2004c). We suspect that recombination is rapid, air plasma ionization is quickly transfered to meteoric metals, and most light originates in the region behind the meteoroid where the plasma has had time to equilibrate to some extend.

One could also suspect that, since the air close to the meteor is strongly ionized, the electrons are strongly coupled to the background geomagnetic field, resulting in strong local electric fields, which, at high altitudes, exert the predominating influence on coupling of the meteor vapor motion to the background air (Zinn, 2003).

However, while the geomagnetic field has been shown relevant to the late evolution of meteor trains (Oppenheim et al., 2003), where it breaks up the train to permit radar reflections at non-specular angles, the field has not been demonstrated to play a role in the immediate wake of the meteoroid, possibly because thermal and non-thermal collisions still dominate the fluid dynamics of the plasma.

\section{Sources of trail broadening}

It has been shown that $50 \%$ of the light emitted by a meteor is recorded in the first $1.1 \times 10^{-4} \mathrm{~s}$ and $90 \%$ after $6 \mathrm{~ms}$ (Jenniskens and Stenbaek-Nielsen, 2004), with an effective exposure time of only about $7 \times 10^{-5} \mathrm{~s}$. After $6 \mathrm{~ms}$, the stars have only moved an angle of $0.09^{\prime \prime}$ and therefore, the train width cannot be influenced by the tracking of the telescope on the stars (instead of the train).

After formation, the cooling air plasma drifts according to the prevailing winds, which can be up to $\sim 100 \mathrm{~m} / \mathrm{s}$ perpendicular to the line of sight. The meteor follows a straight line, but after deposition, the decaying light drifts about 6 pixels $(0.6 \mathrm{~m})$ in $6 \mathrm{~ms}$. That is much less than the observed trail width.

However, the definitive explanation for the wide trail is that the meteor, even at $100 \mathrm{~km}$ altitude, is clearly out of focus when observed with a large telescope. The amount of defocussing $(d)$ can be written as $d=\cos (\phi) *(D f)^{2} / H$, where $\phi$ is the zenith angle $\left(29.8^{\circ}\right.$ for Southern May Ophiuchid meteor) and $H$ the altitude of the object, while $D$ is the diameter of the telescope $(8.2 \mathrm{~m})$, and $f$, the $f$-ratio (focal length/diameter $D$ ) of the Cassegrain focal plane (13.4). This formula gives a defocus of $114 \mathrm{~mm}$ if the object is at an altitude $H$ of $90 \mathrm{~km}$. As the angular defocussing $e$ is expressed by $e=d /\left(f^{2} D\right)$, we have a $0.14^{\prime \prime}$ broadening for each $\mathrm{mm}$ of defocussing. This gives a width of $16.2^{\prime \prime}$ at $H=90 \mathrm{~km}$. This result was checked by ray tracing, which gave $16^{\prime \prime}$ for $H=90 \mathrm{~km}, 14^{\prime \prime}$ for $100 \mathrm{~km}$, and $12^{\prime \prime}$ for $150 \mathrm{~km}$. These values are very close to the measured width of $14^{\prime \prime}$ suggesting that this was the origin of the broadening and consistent with a meteor captured at $100 \mathrm{~km}$ altitude (in agreement also with the expected location of peak brightness).

Unlike our expectations, the trail was probably not resolved and the measured width was only an upper limit. This explains the near constant excitation conditions throughout the emitting volume and the fact that the volume is larger than expected.

\section{Search for carbon atom emission}

Organic matter is expected to be present in some types of meteoroids, but until now it is not known whether that organic matter is fully atomized in the meteor ablation process. Earlier searches of breakup products, such as $\mathrm{CN}$, have not been successful. All carbon atom lines at visible wavelengths have low Einstein A coefficients and are weak. The most intense lines are expected in the near-IR and the far UV. If a significant fraction is atomized, this could result in emission from neutral Carbon atom (C I) lines that can be detected in the relatively unexplored spectral range at longer wavelengths than $900 \mathrm{~nm}$. More importantly, carbon atom emission is also expected from atmospheric $\mathrm{CO}_{2}$, which breaks into its atomic constituents in LTE conditions at $T \sim 4400 \mathrm{~K}$ (Jenniskens et al., 2000). We readily identify strong candidate lines at 905 and $939 \mathrm{~nm}$, but these are about 5 and $2 \mathrm{~nm}$ away from the anticipated emissions of C I (expected at 909.7 and $940.8 \mathrm{~mm}$, respectively), a discrepancy much larger than our wavelength calibration errors. Instead, these lines are identified with atomic emissions from N I at 904.8 and $938.9 \mathrm{~nm}$, respectively.

An upper limit to the total carbon atom abundance in the air plasma is calculated following Jenniskens et al. (2004d). Especially the region from 906 to $910 \mathrm{~nm}$ offers little interference from the sky airglow background. From a comparison of noise at those wavelengths with the intensity of the oxygen $926 \mathrm{~nm}$ lines, we find for the ratio of neutral components: $[\mathrm{C}] /[\mathrm{O}]<2 \times 10^{-4}$.

Most carbon atoms are expected to be in neutral atomic form (like oxygen) with only a small fraction of $\mathrm{C}+\mathrm{We}$ calculated that an LTE air plasma at $4400 \mathrm{~K}$ with the pressure and composition of air at $95 \mathrm{~km}$ altitude would have an expected abundance of $[\mathrm{C}] /[\mathrm{O}]=7 \times 10^{-4}$.

Although this value is no more than $3 \sigma$ higher than our measured upper bound, we conclude that not all atmospheric $\mathrm{CO}_{2}$ is dissociated into $\mathrm{CO}+\mathrm{O}$, and then atomized, as would be expected in LTE conditions and during impacts. This implies that meteors are not as efficient as 
might be expected in aerothermochemistry of an early Earth atmosphere.

Unfortunately, no metal atom line emission is detected in the spectrum that could help relate the upper limit of carbon emission to meteoric ablation products. The expected number density of meteoric vapor in the meteor plasma is about $1.0 \times 10^{-2}$ per $\mathrm{O}$ atom (Boyd, 2000). If so, less than one in a hundred meteoric vapor atoms are carbon. If the ratio of organic refractory mass versus silicate mass is 0.88 (Greenberg, 2000), then the carbon atom abundance should have been about $[\mathrm{C}] /[\mathrm{O}]=4 \times 10^{-3}$, suggesting that perhaps less than 1 in 20 carbon atoms is vaporized in atomic state. Other meteoric carbon may survive the ablation process in the form of small or complex reduced organic molecules or in solid grains, providing potential prebiotic molecules at the time of the origin of life (Jenniskens et al., 2000).

\section{Conclusion}

We conclude that the anomalous signal detected at the $\mathrm{ESO} / \mathrm{VLT}$ was a meteor of brightness $-7.8 \pm 1.1$ magnitude. The variation of spectral features across the slit shows that the ratio of atomic and molecular nitrogen emission appeared to be constant in the whole volume of the emitting plasma. We conclude that this is mainly due to the fact that the trail is out of focus, a problem that will persist in future observations of meteor trails with large telescopes focused at infinity. From the lack of carbon atom emission in the spectrum, we conclude that the air plasma did not efficiently dissociate atmospheric $\mathrm{CO}_{2}$ into atomic carbon at the level expected for LTE conditions, but our measured upper abundance of neutral carbon atoms is only $3 \sigma$ below that expected.

\section{Acknowledgements}

The raw data of this serendipitous observation were kindly made available for analysis by Dr. C. Lidman (ESO) on behalf of the SCP collaboration. We thank the anonymous referees for the helpful comments for improving this paper.

\section{References}

Baggaley, W.J. The determination of the initial radii of meteor trains. Mont. Not. R. Astron. Soc. 147, 231-243, 1970.

Baggaley, W.J. Measurements of the velocity dependence of the initial radii of meteor trains. Bull. Astron. Inst. Czech. 31, 308-311, 1980.

Baggaley, W.J. Single wavelength measurements of the initial radii of radio meteor ionization columns. Bull. Astron. Inst. Czech. 32, 345 349, 1981.

Boyd, I.D. Computation of atmospheric entry flow about a Leonid meteoroid. Earth Moon Planets 82-83, 93-108, 2000.

Ceplecha, Z., Borovicka, J., Elford, W.G., Revelle, D.O., Hawkes, R.L., Porubcan, V., Šimek, M. Meteor phenomena and bodies. Space Sci. Rev. 84, 327-471, 1998.

Cook II, A.F., Hawkins, G.S., Steinon, F.M. The width of meteor trails II. Astron. J. 64, 327, 1959.

Cook, A.F., Hawkins, G.S., Stienon, F.M. Meteor trail widths. Astron. J. 67, 158-162, 1962.

Greenberg, J.M. From comets to meteors. Earth Moon Planets 82-83, 313-324, 2000.

Jenniskens, P., Laux, C.O., Packan, D.M., Wilson, M., Fonda, M. Meteors as a vector for the delivery of organic matter to the early Earth. Earth Moon Planets 82-83, 57-70, 2000.

Jenniskens, P., Jehin, E., Cabanac, R.A., Laux, C.O., Boyd, D.I. Spectroscopic anatomy of a meteor trail cross section with the European Southern Observatory Very Large Telescope. Meteorit. Planet. Sci. 39 (4), 1-8, 2004a.

Jenniskens, P., Laux, C.O., Wilson, M., Schaller, E. The mass and speed dependence of meteor plasma temperatures. Astrobiology 4 (1), 81-94, $2004 b$

Jenniskens, P., Laux, C.O., Schaller, E.L. Search for the OH(X2P) Meinel band emission in meteors as a tracer of mineral water in comets: detection of $\mathrm{N}_{2}+(\mathrm{A}-\mathrm{X})$. Astrobiology 4 (1), 109-121, 2004c.

Jenniskens, P., Schaller, E.L., Laux, C.O., Schmidt, G., Rairden, R.L. Meteors do not break exogenous organic molecules into di-atoms. Astrobiology 4 (1), 67-79, 2004d.

Jenniskens, P., Stenbaek-Nielsen, H.C. Meteor wake in high frame-rate images and implications for the thermal processing of ablated organic compounds in air. Astrobiology 4 (1), 95-108, 2004.

Öpik, E.J. Physics of Meteor Flight in the Atmosphere. Interscience Publishing, New York, 174 pp, 1958.

Oppenheim, Meers M., Dyrud, Lars P., vom Endt, Axel F. Plasma instabilities in meteor trails: 2-D simulation studies. J. Geophys. Res. (Space Phys.) 108 (A2), SIA 8-1, 2003.

Stenbaek-Nielsen, H.C., Jenniskens, P. A "shocking" Leonid meteor at 1000 fps. Adv. Space Res. 33, 1459-1465, 2004.

Zinn, J. The 1000-fps Alaska Leonid - Computations and speculations. Proceedings of the 2003 Leonid MAC Workshop and Science Meeting, NASA Ames Research Lab, August 2003.

Zinn, J., Judd, O.P., ReVelle, D.O. Leonid meteor ablation, energy exchange, and trail morphology. Adv. Space Res. 33, 1466-1474 (and Erratum to same, Adv. Space Res., 34, 1839), 2004. 